\title{
THE ANTI-CYCLONIC SHEAR WAVE: A NEW GEOPHYSICAL WAVE
}

\author{
ROGER L. HUGHES ${ }^{1}$
}

(Received 16 July 1982; revised 12 December 1982)

\begin{abstract}
A new type of first baroclinic mode wave which propagates on an anti-cyclonic vorticity field is identified. It is of the vorticity class of waves which contains Rossby waves amongst others. This anti-cyclonic shear wave is produced by pressure variations distorting the vertical stratification in such a manner that the associated vortex stretching generates the velocity variation required for Bernoulli compatibility with the initial pressure variation. The wave travels at a speed characteristic of particles within the undisturbed shear flow and is a low frequency and low wavenumber wave. In the present study this wave is considered in the presence of a weak anti-cyclonic shear.
\end{abstract}

\section{Introduction}

It is well known in fluid dynamics that waves can propagate on the gradient of a quantity appropriately related to the vorticity. Examples of such waves in geophysical fluid dynamics are:

(i) Horizontal shear waves which propagate on the gradient of the vorticity of the flow.

(ii) Rossby (or planetary) waves which propagate on the gradient of background vorticity associated with the earth's rotation.

(iii) Shelf (and trench) waves which propagate on the gradient of potential vorticity associated with depth variations.

In a strict mathematical sense all these waves are of the same class. A system consisting of a spatially varying shear in a flow over variable bottom topography in the presence of a differentially rotating environment will exhibit a single set of

\footnotetext{
'A.N.M.R.C., P.O. Box 5089AA, Melbourne, Victoria 3001. Present address: Department of Geology and Geophysics, Yale University, New Haven, CT 06511, U.S.A.

(c) Copyright Australian Mathematical Society 1983
} 
shear-related waves which are hybrid of the above three types. It is impossible to identify a particular wave with a particular shear-related process unless one process dominates all others. The purpose of this study is to introduce another member of this class of waves which propagates not on the gradient of a quantity related to the vorticity but on the vorticity itself, provided the vorticity is anti-cyclonic.

To understand how this new wave fits into the vorticity class of waves, consider the simple vorticity equation for a spatially unbounded ocean in the reference frame moving with the phase velocity of a hypothesised wave of the vorticity class. The equation governing the resulting flow is commonly of the form

$$
\nabla^{2} \psi=\eta_{1}(\psi)+\eta_{2}(\psi, y)+\eta_{3}(\psi, x, y)=\eta,
$$

where $\nabla^{2}$ is the horizontal Laplacian operator in co-ordinates $(x, y), \psi$ is a velocity stream-function for nearly non-divergent flow and $\eta$ is the vorticity. The vorticity is composed of three parts $\left(\eta_{1}, \eta_{2}\right.$ and $\left.\eta_{3}\right)$ which respectively represent the basic vorticity, the background vorticity and the (small) topographically induced vorticity. The hypothesized wave can exist only if $d \eta / d \psi<0$ in some region in which (1.1) admits a sinusoidal solution in both $x$ and $y$.

However, Hughes [3] has shown that if allowance is made for vortex stretching in response to Bernoulli induced pressure variations, the stagnation vorticity

$$
\nabla^{2} \psi+\left(f / 2 c^{2}\right)(\nabla \psi)^{2}
$$

is conserved following a fluid particle in a baroclinic ocean with a single active layer overlying a passive layer. Here $\nabla$ is the horizontal gradient operator, $f$ is the constant Coriolis parameter and $c$ is a positive constant equal to the celerity (speed of a Kelvin wave). (Allowance for variation in $c$ with $\psi$, although real, is largely cosmetic.) It follows that in such a baroclinic ocean a more appropriate form than $(1.1)$ is

$$
\nabla^{2} \psi=\eta-\left(f / 2 c^{2}\right)(\nabla \psi)^{2}
$$

By substituting $\xi=\exp \left(f \psi / 2 c^{2}\right)$ into this equation, the right hand side reverts to being purely algebraic with no differential operator. The resultant equation may admit a fully sinusoidal wave only if

$$
\frac{d \eta}{d \psi}+\frac{f}{2 c^{2}} \eta<0 .
$$

Even if $d \eta / d \psi$ is zero or positive, waves may possibly exist if $f \eta<0$. Thus even when $\eta$ is increasing in $\psi$ there is a possibility of waves if the stagnation vorticity is anti-cyclonic.

It is interesting to note that other models exist in which there is no gradient of vorticity (or potential vorticity) except at a finite number of isolated points. The models of Niiler and Mysak [5] and Collings and Grimshaw [2] are good 
examples. These studies consider the dispersion relation for waves propagating on various basic flows with piecewise constant potential vorticity. However, because of the finite number of jumps of potential vorticity in these models, these models cannot be regarded as totally free of potential vorticity gradients. The present model contains no such jumps (or any other form of variation) in either the vorticity or topographic structure. However, the waves themselves are able to generate the gradient of vorticity necessary for their propagation. The model's form has been chosen so that it does not admit waves of types (i)-(iii) above. Furthermore, by using the simple formulation used to obtain (1.3), other waves such as Kelvin and Poincaré waves have been filtered from the model. However, the model exhibits a wave which is governed by the anti-cyclonic vorticity of the basic flow in confirmation of the ideas expressed above. Unlike the studies of Niiler and Mysak [5] and Collings and Grimshaw [2] the stability of the flow is not considered here, that is, only non-growing waves are studied.
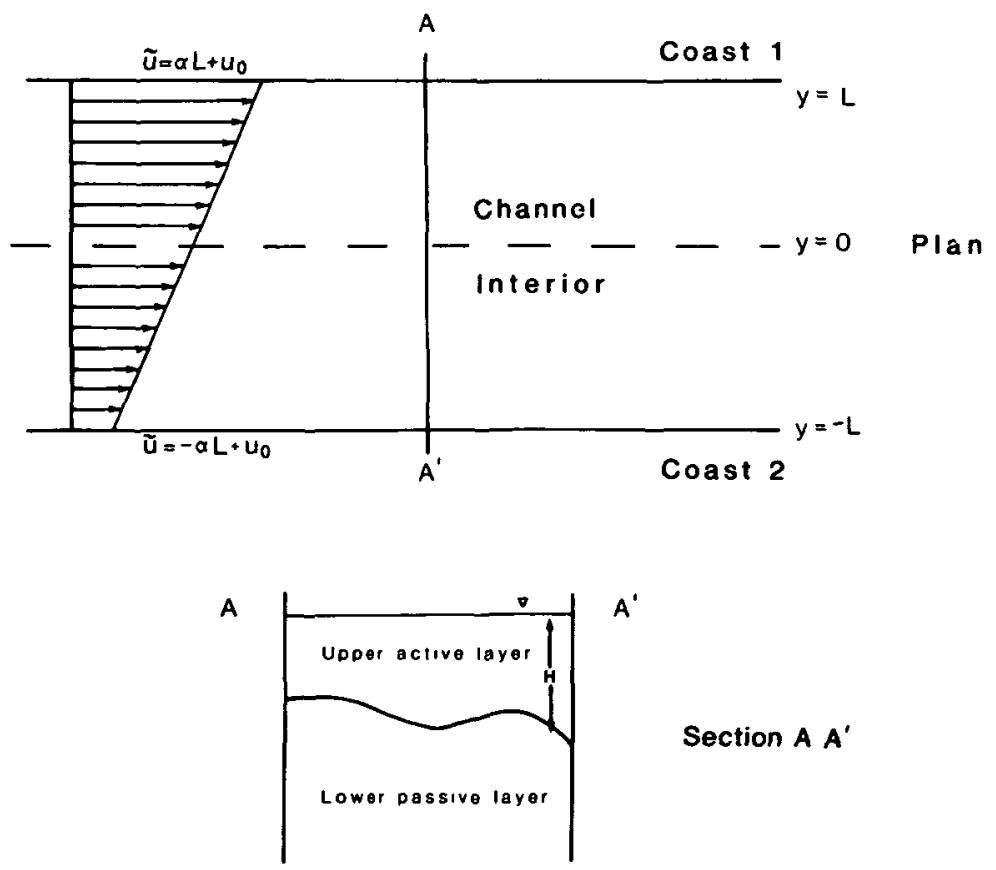

Section A A'

Figure 1. Diagrammatic representation of the model used which consists of a two layer flow within an infinitely long channel. The upper layer has a shear of $\alpha$, with free slip boundaries. 


\section{The governing equation}

Consider the model shown in Figure 1. This model consists of a two layer frictionless hydrostatic reduced gravity ocean. The lower (half) layer is deep and passive, while the upper layer is active and contains a laterally sheared basic flow with constant shear $\alpha$ and mean speed $u_{0}$. All motion is on an $f$-plane. We define two Cartesian co-ordinate systems $(\tilde{x}, \tilde{y})$ and $(x, y)$. The former represents a system such that the lower layer is at rest. The latter represents a system in which a travelling wave of permanent form appears steady. Thus,

$$
\left.\begin{array}{l}
x=\tilde{x}-c_{s} t \\
y=\tilde{y}
\end{array}\right\}
$$

where $c_{s}$ is the phase speed of the wave and $t$ is time. For mathematical convenience, we restrict attention to flows in which variations in the upper layer depth, $\delta H$, are small compared to the overall depth of the layer, $H$, that is $|\delta H / H| \ll 1$, which may be shown to require

$$
\left|u_{0} f L\right|^{1 / 2}, \quad\left|c_{s}\right|, \quad|\alpha L| \text { and }\left|u_{0}\right| \text { all } \ll c .
$$

It follows that continuity can be used to define an upper layer velocity streamfunction according to

$$
u=-\frac{\partial \psi}{\partial y}, \quad v=\frac{\partial \psi}{\partial x},
$$

where $(u, v)$ represents the steady velocity field corresponding to the Cartesian co-ordinates $(x, y)$. No assumption based upon geostrophy is required.

As noted in the previous section (equation (1.2), but with a modification for the speed of the lower layer as required by this frame of reference), the quantity

$$
\nabla^{2} \psi+\left(f / 2 c^{2}\right)(\nabla \psi)^{2}+\left(f^{2} c_{s} / c^{2}\right) y
$$

is conserved following a fluid particle in steady motion with weak shear, $(\alpha / f) \ll$ 1. For the linear shear flow shown in Figure 1,

$$
\begin{array}{r}
\nabla^{2} \psi+\left(f / 2 c^{2}\right)(\nabla \psi)^{2}+\left(f^{2} c_{s} / c^{2}\right) y=-\alpha+\left(f / 2 c^{2}\right)\left(-2 \alpha \psi+\left(u_{0}-c_{s}\right)^{2}\right) \\
+\left(f^{2} c_{s} / \alpha c^{2}\right)\left(-\left(u_{0}-c_{s}\right) \pm\left(\left(u_{0}-c_{s}\right)^{2}-2 \alpha \psi\right)^{1 / 2}\right),
\end{array}
$$

where the \pm choice depends on the sign of $\left(u_{0}-c_{s}\right)$ and where

$$
\psi=-\frac{1}{2} \alpha y^{2}-\left(u_{0}-c_{s}\right) y .
$$

As the right hand side of (2.5) involves only $\psi$ which, like the stagnation vorticity on the left hand side, is conserved following a particle, (2.5) can be used to study 
the behaviour of the system when a wave is present. The boundary conditions applicable to such a study are

$$
\psi=-\frac{1}{2} \alpha L^{2}-\left(u_{0}-c_{s}\right) L \text { at } y=L,
$$

and

$$
\psi=-\frac{1}{2} \alpha L^{2}+\left(u_{0}-c_{s}\right) L \text { as } y=-L .
$$

This is equivalent to specifying that there be no flux across ithe boundaries and rou net transportation of water by the wave. Unlike in the classical Eady problem, which shares much in common with the present analysis, there is no specification of a no-slip condition at the boundaries for the basic flow.

Perturbing (2.5) about the basic solution (2.6) gives an equation for the wave induced perturbation $\psi^{\prime}$ in $\psi$. This equation is

$$
\begin{aligned}
\nabla^{2} \psi^{\prime}+ & \frac{f}{c^{2}}\left(-\alpha y-\left(u_{0}-c_{s}\right)\right) \frac{d \psi^{\prime}}{d y} \\
& =-\frac{f}{c^{2}} \alpha \psi^{\prime} \mp \frac{f^{2} c_{s}}{c^{2}} \frac{\psi^{\prime}}{\left(\left(u_{0}-c_{s}\right)^{2}+\alpha^{2} y^{2}+2 \alpha\left(u_{0}-c_{s}\right) y\right)^{1 / 2}} .
\end{aligned}
$$

There is no need to perturb the functional form of the right hand side. Such a perturbation might be interpreted as a change in the form of the basic shear flow. When $\alpha \equiv 0,(2.8)$ reduces to

$$
\nabla^{2} \psi^{\prime}-\frac{f}{c^{2}}\left(u_{0}-c_{s}\right) \frac{d \psi^{\prime}}{d y}+\frac{f^{2} c_{s}}{c^{2}\left(u_{0}-c_{s}\right)} \psi^{\prime}=0 .
$$

The \pm choice associated with the sign of $\left(u_{0}-c_{s}\right)$ is absorbed by noting $\pm\left(\left(u_{0}-c_{s}\right)^{2}\right)^{1 / 2}=\left(u_{0}-c_{s}\right)$.

Substituting

$$
\psi^{\prime}=\operatorname{Im} \exp (i l(y+L)) \sin (k x),
$$

where $\operatorname{Re}(l)=N \pi / 2 L$ with $N$ an integer, gives

$$
\left(N \frac{\pi}{L}\right)^{2}+\frac{f^{2}}{c^{4}}\left(u_{0}-c_{s}\right)^{2}+4 k^{2}=\frac{4 f^{2} c_{s}}{c^{2}\left(u_{0}-c_{s}\right)} .
$$

As the left hand side consists of the sum of three real squares and therefore is positive, the right hand side must also be positive. Thus $c_{s} / u_{0}$ is positive and less than unity. For large $|k c / f|, c_{s}$ tends to $u_{0}$, that is, the wave is advected by the surface flow. For smaller $|k c / f|$ the wave moves at a speed less than the speed of the upper layer, although it can never be stationary. For $u_{0} \equiv 0$ no wave exists. These waves have an evanescent across-channel structure and are restricted to situations containing a boundary. The waves are propagating on the across-channel depth variation of the stratification as produced by the difference in the 
along-channel velocity of the two layers. This depth variation functions in a very similar manner to a total depth variation on which topographic shelf waves can propagate. The present study is not concerned with such coastal waves. These coastal waves may be filtered from the system by insisting that

$$
\left(\frac{f}{\alpha}\right) \cdot\left(\frac{c_{s}}{\alpha L}\right) \ll 1 .
$$

In this situation (2.5) and (2.8) reduce to

$$
\nabla^{2} \psi+\frac{f}{2 c^{2}}(\nabla \psi)^{2}=-\alpha+\frac{f}{2 c^{2}}\left(-2 \alpha \psi+\left(u_{0}-c_{s}\right)^{2}\right),
$$

and

$$
\nabla^{2} \psi^{\prime}+\frac{f}{c^{2}}\left(-\alpha y-\left(u_{0}-c_{s}\right)\right) \frac{d \psi^{\prime}}{d y}=-\frac{f}{c^{2}} \alpha \psi^{\prime},
$$

respectively. Writing

$$
\psi^{\prime}=\psi^{\prime \prime} \sin (k x)
$$

where $k$ is the wavenumber, (2.14) implies

$$
\frac{d^{2} \psi^{\prime \prime}}{d Y^{2}}-s\left(Y+P_{1}\right) \frac{d \psi^{\prime \prime}}{d Y}=-s P_{2} \psi^{\prime \prime},
$$

where the following non-dimensional quantities have been used:

$$
\begin{aligned}
s & =\operatorname{sgn}(\alpha f), \\
Y & =y \frac{(s f \alpha)^{1 / 2}}{c}, \\
P_{1} & =\frac{(s f \alpha)^{1 / 2}}{\alpha c}\left(u_{0}-c_{s}\right), \\
P_{2} & =1-\frac{c^{2}}{f \alpha} k^{2}=1-P_{3} .
\end{aligned}
$$

The boundary conditions, (2.7), perturb to $\psi^{\prime \prime}=0$ at $y= \pm L$. It follows that for $\psi^{\prime \prime} \neq 0$, there must be at least one maximum or minimum of $\psi^{\prime \prime}$ in the range $-L<y<L$. At such a maximum or minimum both $\left(d^{2} \psi^{\prime \prime} / d Y^{2}\right) / \psi^{\prime \prime} \leqslant 0$ and $d \psi^{\prime \prime} / d Y=0$ by definition of such an extremum. Thus (2.16) implies $s P_{2} \geqslant 0$. The case $s=0$ was covered by (2.9).

If $s<0,(2.17)$ implies $P_{2} \geqslant 0$ for all $k$. If $s>0,(2.17)$ implies $P_{2}>0$ for all $k$ but $P_{2}<0$ for $k^{2}>f \alpha / c^{2}$. It follows that the condition $s P_{2} \geqslant 0$ is satisfied only if both

(i) $s>0$, that is, the flow is anti-cyclonic, and

(ii) $k^{2} \leqslant f \alpha / c^{2}$, that is, the waves are long. 
Both of these features are distinctive of the type of wave being studied here. The author is unaware of any mention of such waves in the literature.

One further property of the wave is deducible without solving the governing equations. Relative to a frame of reference moving at a speed of $u_{0}$, the flow in Figure 1 is unaffected by rotating the figure by $180^{\circ}$ provided interfacial position variations caused by the flow in the lower layer are ignored. It follows that there is a basic symmetry to this $f$-plane system which requiires the dispersion reiation to be of the form

$$
\omega / k-u_{0}=c_{s}-u_{0}=\mathrm{fn}(|k|),
$$

where $\omega$ is the wave frequency, and $\mathrm{fn}$ is a function to be determined from the governing equations. Notice that the far right hand side of (2.18) is independent of the sign of $k$. As $u_{0}$ merely Doppler shifts the frequency, $u_{0}$ is taken to be zero, unless stated otherwise, in what follows.

\section{The solution for a modified basic flow}

The basic equation governing the flow in the presence of a wave, (2.13), is non-linear and it is difficult to appreciate except in its perturbed form, (2.14). The equation may be made more tractable by noting that the wave which we wish to consider propagates on the shear of the flow velocity itself. This suggests replacing $\psi$ on the right hand side of (2.13) by $\Phi$ where

$$
\Phi=\frac{2 c^{2}}{f}\left(1-\exp \left(-\frac{f}{2 c^{2}} \psi\right)\right) .
$$

Note that $\psi=0$ implies $\Phi=0$ and $d \Phi / d \psi=1$. This approximation is purely heuristic. However, the resulting equation is that which would result if the basic flow were given by the stream -function

$$
\psi=\frac{2 c^{2}}{f} \ln \left(\frac{2}{3}+\frac{\cos \left(\left(3 f \alpha / 2 c^{2}\right)^{1 / 2} y\right)}{\cos \left(\left(3 f \alpha / 2 c^{2}\right)^{1 / 2} L\right)}\left(\exp \left(\frac{-f}{4 c^{2}} \alpha L^{2}\right)-\frac{2}{3}\right)\right),
$$

under the slow wave conditions (2.2) and (2.12). It may be simpler to interpret the new equation resulting from (3.1) as resulting from such a flow rather than a heuristic approximation. This flow is physically unacceptable for small values of $\cos \left(\left(3 f \alpha / 2 c^{2}\right)^{1 / 2} L\right)$ as it contains vortex sheets. For

$$
\left(3 f \alpha / 2 c^{2}\right)^{1 / 2} L>\pi
$$

the flow contains internal flow reversals other than that at $y=0$. Such flows are of little interest here. 
Replacing $\psi$ by $\Phi$ on the right hand side of (2.5) and using the substitution

$$
\xi=\exp \left(\frac{f \psi}{2 c^{2}}\right)
$$

implies the Helmholtz equation

$$
\nabla^{2} \xi=\frac{f}{2 c^{2}}\left(\left(-3 \alpha+\frac{f}{2 c^{2}} c_{s}^{2}\right) \xi+2 \alpha\right) .
$$

As this equation is linear, any solution satisfying the corresponding homogeneous equation and homogeneous boundary condition represents a free disturbance to the system. A free disturbance of (3.5) in the form of a wave satisfies

$$
-\left(k^{2}+l^{2}\right)=\frac{f}{2 c^{2}}\left(-3 \alpha+\frac{f}{2 c^{2}} c_{s}^{2}\right),
$$

where $(k, l)$ is the wavenumber of the disturbance such that

$$
l=N \pi / 2 L
$$

for integer $N$. However, for the present, this heuristic model is only used with $N=1$. (This being the requirement for (3.3) to be satisfied.) As $c_{s}=\omega / k$ by definition of phase velocity in the co-ordinate system $(\tilde{x}, \tilde{y}),(3.6)$ implies the dispersion relation

$$
\omega= \pm k\left(\frac{2 c^{2}}{f}\left(3 \alpha-\frac{2 c^{2}}{f}\left(k^{2}+l^{2}\right)\right)\right)^{1 / 2}
$$

Figure 2 illustrates this dispersion relation for different values of

$$
\lambda=\frac{1}{f}\left(3 \alpha-\frac{2 c^{2}}{f} l^{2}\right) .
$$

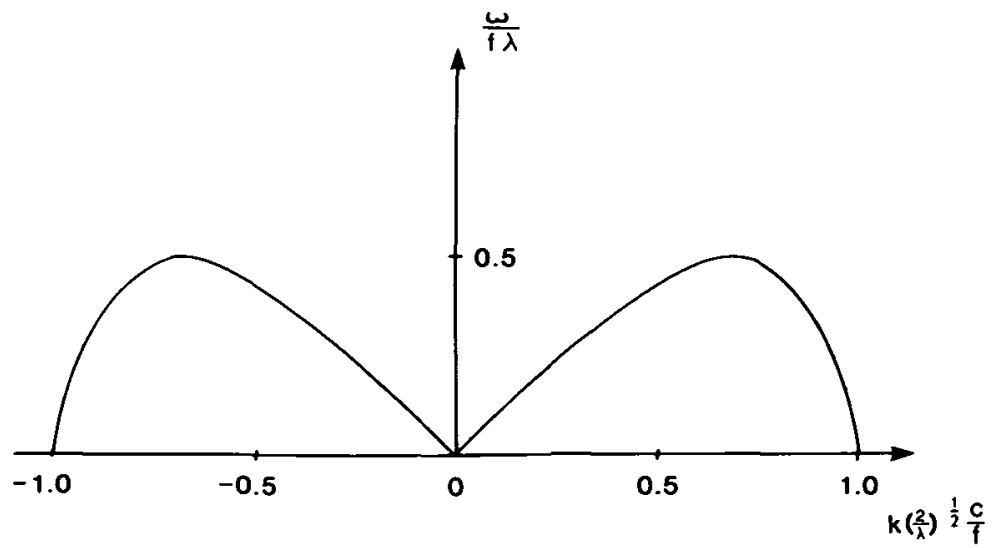

Figure 2. The approximate dispersion relation generated by making the simple heuristic change to the basic equation. The model is as shown in Figure 1. 
A wave is only possible if $\lambda>0$. This requires

$$
\alpha f>\frac{2}{3} c^{2} l^{2} .
$$

Thus the basic shear upon which the wave propagates must be anti-cyclonic as expected. Figure 2 shows that the wave is a low wavenumber wave with

$$
k^{2}<\frac{1}{2}(f / c)^{2} \lambda
$$

This type of behaviour is similar to that predicted in the previous section. The dispersion relation is only applicable to the small wave speeds as given by (2.12).

Figure 3 shows diagrammatically the form of the perturbation streamfunction which is attributable to the wave. The wave appears as a sequence of alternating cyclonic and anti-cyclonic cells superimposed on the basic shear flow. Near the boundaries $y= \pm L$ of a cyclonic cell, the flow is against that of the basic shear flow. This reduction in speed of particles constituting the shear flow implies that the pressure (by Bernoulli's equation) and hence the depth of the upper layer (by hydrostatic balance) increase. The resulting vortex stretching generates the cyclonic vorticity required by the cell. Near the boundaries $y= \pm L$ of an anti-cyclonic cell, the speed of the basic shear flow is increased by the presence of the wave. This causes a decrease in upper layer depth and generates the anti-cyclonic vorticity required by the cell. A wave is not possible in a cyclonic shear because the perturbations to the basic shear flow are not consistent with the type of vorticity required by vortex stretching or compression. Notice that it is essential for the phase velocity of the wave to be equal to the velocity of some particle in the undisturbed shear flow, so that the required vorticity is generated near both boundaries.

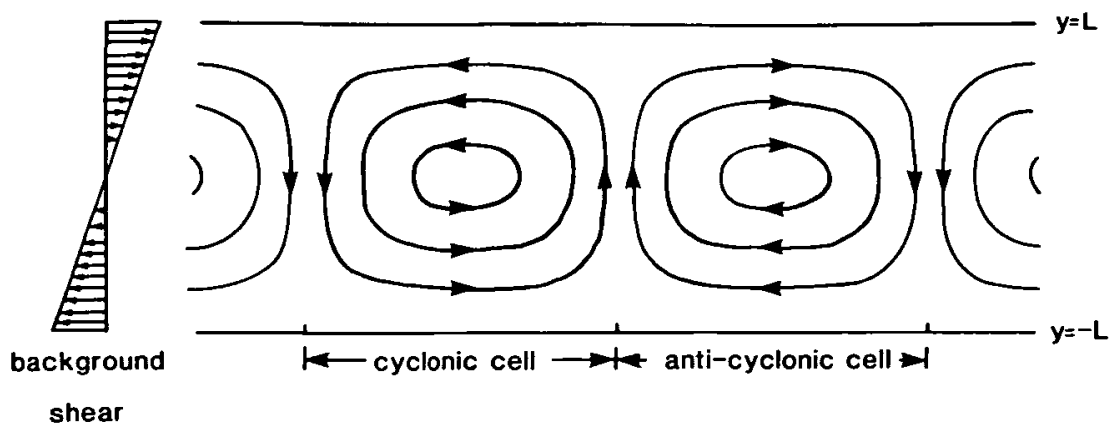

Figure 3. Diagrammatic representation of the cellular structure of an anti-cyclonic shear wave. 


\section{Small amplitude waves}

Having used a simple change to the governing equation, (2.13), to obtain a qualitative description of the wave being studied, it is necessary to study the actual governing equation more closely. In this section we consider a small amplitude wave, the cross-channel structure of which is governed by (2.16). This equation is Hermite's equation and has polynomial solutions for negative integer $P_{2}$ [1]. For more general $P_{2}$ the solution involves a parabolic cylinder function times a decaying Gaussian function all centered on $Y=-P_{1}$. However, it is tedious to fit this solution to the boundary conditions. Given the understanding of the form of the dispersion relation from the previous section a numerical solution is more appropriate.

In obtaining a numerical solution, it is easiest to integrate (2.16) from the boundary at $Y=-Y_{B}=-L(f \alpha)^{1 / 2} / c$ to the other boundary at $Y=Y_{B}$. To achieve this, (2.16) is written as

$$
\frac{d^{2} \psi^{\prime \prime}}{d Y^{2}}-\left(\left(Y+Y_{B}\right)+P_{4}-\frac{(f / \alpha)^{1 / 2}}{c} c_{s}\right) \frac{d \psi^{\prime \prime}}{d Y}=-P_{2} \psi^{\prime \prime},
$$

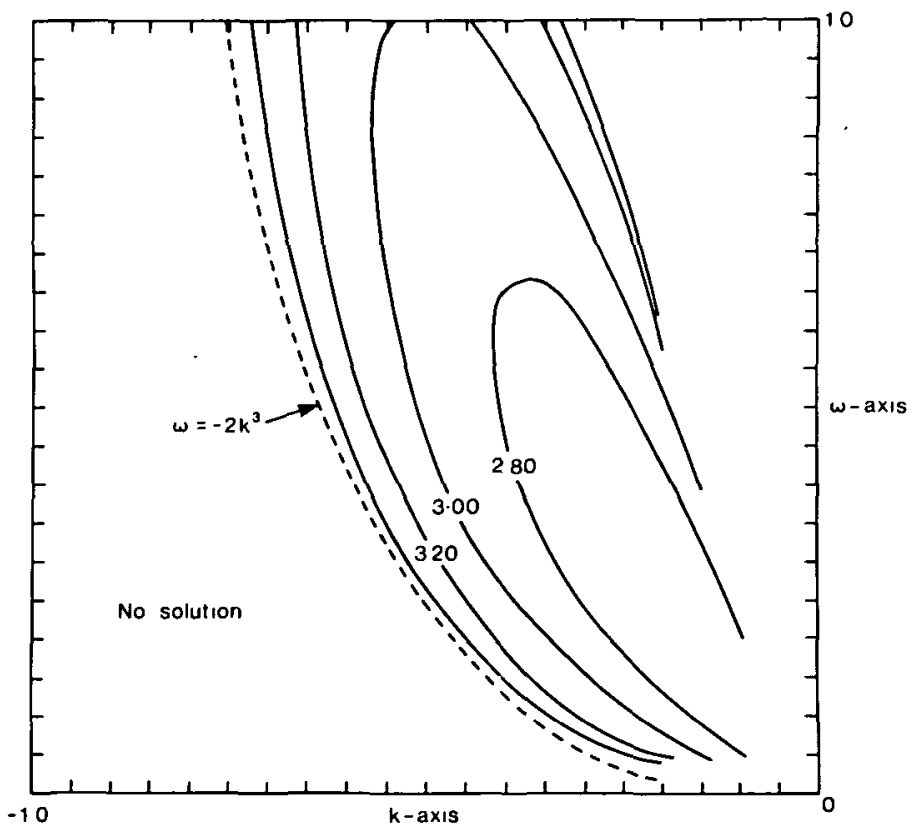

Figure 4. Non-dimensional representation of the dispersion relation for small amplitude anti-cyclonic shear waves in the channel of Figure 1. The frame of reference is moving with the flow at $y=-L$. Curves are for different values of $\gamma=2 L(\alpha f)^{1 / 2} c$. The non-dimensional curve $\omega=-2 k^{3}$ is an approximation for large $\gamma$ and intermediate $\omega$. 
where, retaining $u_{0}$,

$$
P_{4}=\frac{(f / \alpha)^{1 / 2}}{c} u_{0}-Y_{B}
$$

Taking the velocity of the shear flow at the edge of the channel $\left(Y=-Y_{B}\right)$, rather than at the channel centre, as the reference velocity gives $P_{4}=0$. With this new reference velocity, (4.1) was numerically integrated using a Runge-Kutta procedure, across the channel to obtain the dispersion relation given by Figure 4 . Non-dimensionalization was achieved by plotting $\omega / \alpha$ as a function of $k c /(\alpha f)^{1 / 2}$. The plot is restricted to those solutions for which $\omega / \alpha>0$. The dispersion relation shown appears to have little relationship to that calculated in the previous section. However, the dispersion relation has been Doppler shifted according to the variation in velocity between the shear flow at the channel centre and that at the channel wall $\left(Y=-Y_{B}\right)$. This correction depends on both the wavenumber and the channel width. Making the correction in the standard way gives the dispersion relation shown in Figure 5. The dispersion relation is now of very similar form to the qualitative description available from Figure 2. The large effect of the Doppler shift emphasises that the wave travels with a phase velocity equal to the speed of some particle in the undisturbed shear flow. The disturbance could loosely be described as being advected at this speed.

The group velocity of the wave being studied is in the same direction as the phase velocity for very small wavenumbers. For intermediate wavenumbers (less than the maximum wavenumber, $k_{M}$ ) the group and phase velocities are in opposite directions. With the possible exception of the limit on $k$ at $k_{M}$, this type

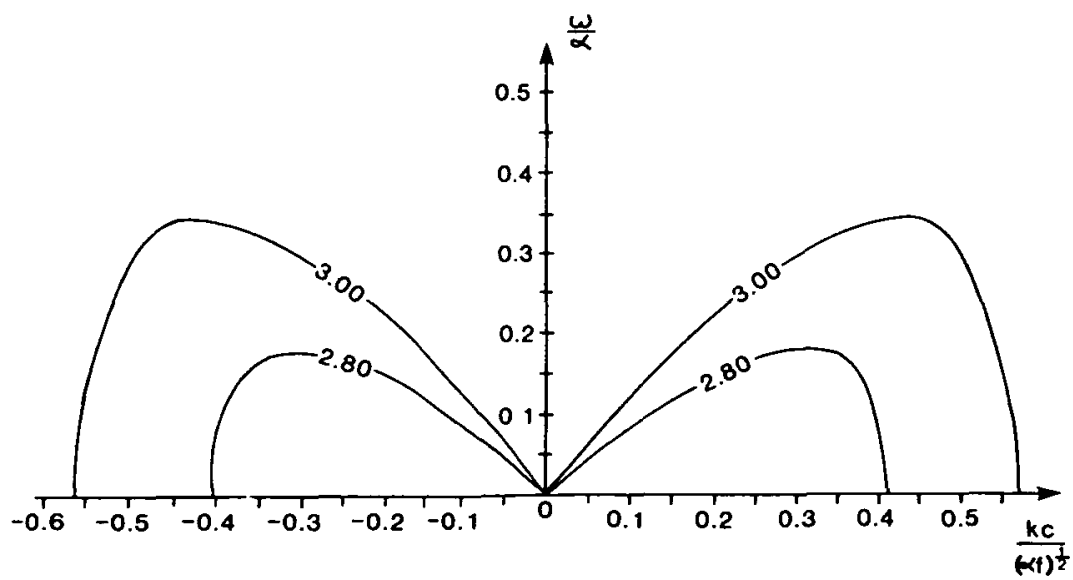

Figure 5. Dispersion relation for small amplitude anti-cyclonic shear waves in the channel of Figure 1. The frame of reference is moving with the flow at $y=0$. Curves are for different values of $\gamma$. Compare this figure with Figure 2. 
of behaviour is similar to that of other members of this class of waves such as Rossby waves. At $k=k_{M}$, the group velocity appears to be unbounded. This unboundedness is in contradiction with common experience which limits its magnitude to a maximum value of $c$. The dilemma is easily resolved by noticing that in non-dimensional units a speed of $c$ appears as a slope of

$$
c \frac{(\alpha f)^{1 / 2}}{\alpha c}=\left(\frac{f}{\alpha}\right)^{1 / 2} \gg 1 .
$$

Strictly, the model is only valid in the limit as $(f / \alpha)^{1 / 2}$ tends to infinity. Hence the appearance of an infinite slope in Figure 5 should be interpreted as implying a group velocity of the order of $\pm c$ as appropriate.

The similarity of the present model to that of the classical Eady problem for baroclinic instability (which also has a constant vorticity, except at the boundaries) suggests that wavenumbers greater than $k_{m}$ may be unstable. However, the present model is not applicable to growing waves because it requires a steady flow in the frame of reference of the wave phase.

Figures 6 and 7 show the structure of the wave amplitude across the channel for various values of $\left(\omega / \alpha, k c /(\alpha f)^{1 / 2}\right)$ normalized so that $d \psi^{\prime \prime} / d Y=1$ at $Y=-Y_{B}$. Those structures shown in Figure 6 correspond to the wave being concentrated on the boundary $Y=-Y_{B}$, while those structures shown in Figure 7

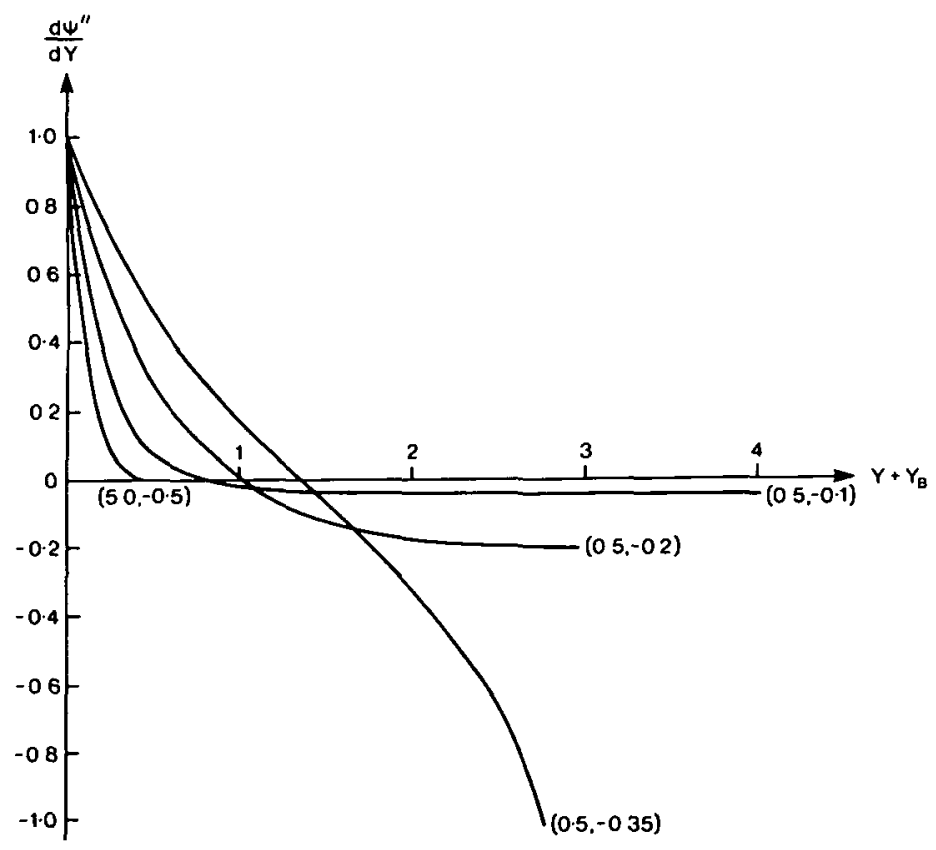

Figure 6. Velocity amplitude structure for various waves concentrated on the boundary at $y=-L$. 
are concentrated on the boundary $Y=Y_{B}$. The wave appears to be concentrated on the boundary at which

$$
Y=Y_{B} \cdot \operatorname{sgn}(\text { phase velocity). }
$$

Thus the wave is concentrated on the boundary for which the background shear flow is in the same direction as the phase velocity. This provides further indication of the strong role of advection in governing the behaviour of the wave.

As this wave is apparently boundary trapped for large values of $L(f \alpha)^{1 / 2} / c$, it might be expected that it represent a modification to a low frequency Kelvin wave by the weak anti-cyclonic shear. To investigate this we consider a wave with a frequency much larger than $\alpha$. Figure 6 shows the structure of such a wave with $\omega / \alpha=5$. This wave is strongly confined to the boundary, $Y=-Y_{B}$. However, this boundary is not that appropriate to a Kelvin wave travelling in the same direction. It follows that the wave is unlikely to be a direct modification of a Kelvin wave. Any Kelvin wave has been filtered out of the present study because, unless it is stalled (which on the above appears not to be so), the speed is of order $\pm c$ and hence too fast for the non-divergent assumption used before in defining the stream-function, $\psi$, to be valid. It should be noted that, for large $L(f \alpha)^{1 / 2} c$, condition (2.12) is violated and the anti-cyclonic shear wave merges with the

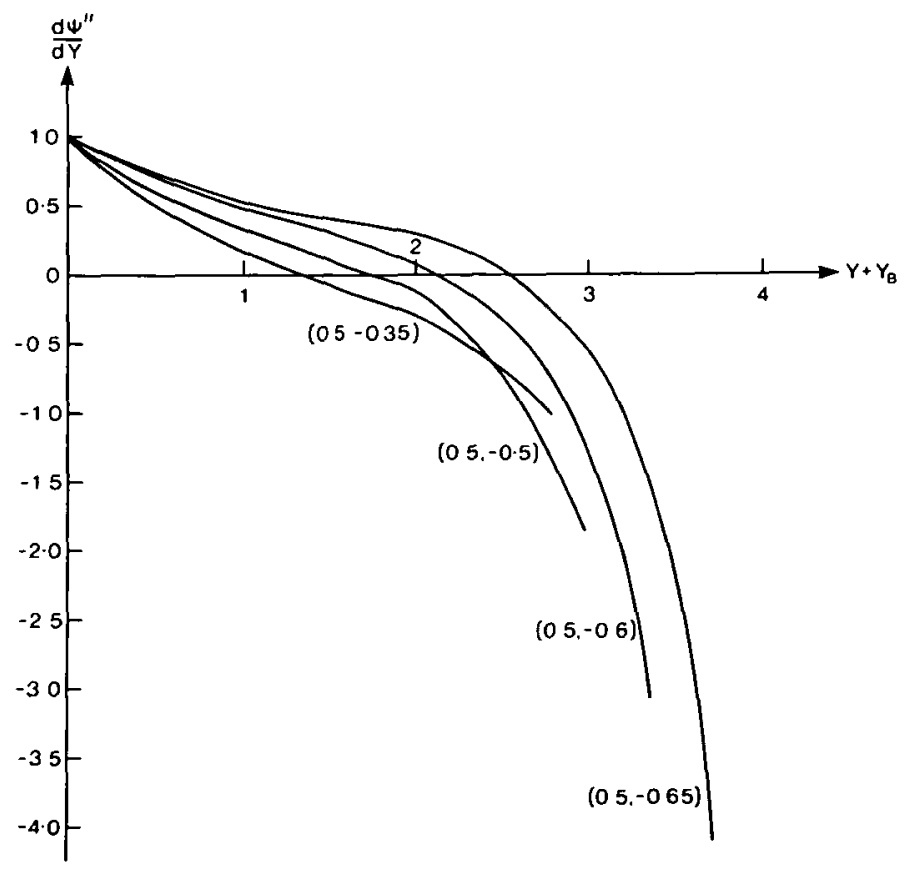

Figure 7. As for Figure 6 but waves concentrated on the boundary $y=L$. 
coastal wave described by the dispersion relation (2.11) (with a modification for the shear). However no such difficulty is encountered if the model is redefined by specifying the wave to be stationary (so that $c_{s}=0$ ) against a background flow with $u_{0} \neq 0$. Then an "apparent wave frequency" may be defined as

$$
\omega=-k u_{0},
$$

and the "apparent dispersion relation" given in Figure 5 is valid for all $L(f \alpha)^{1 / 2} / c$.

\section{Role in mid-ocean}

So far the anti-cyclonic shear wave has been studied in a channel only. However, the restriction to a channel is not essential to the mechanism outlined at the end of Section 3. The purpose of this Section is to give an example with different boundary conditions and note the possible importance of the wave to the mid-ocean. For this purpose we consider a broad anti-cyclonic region embedded in a stationary ocean as shown in Figure 8.

In the regions adjacent to the anti-cyclonic zone, the appropriate equation which is equivalent to (2.16) may be shown to be

$$
\frac{d^{2} \psi^{\prime \prime}}{d Y^{2}}-P_{1} \frac{d \psi^{\prime \prime}}{d Y}-P_{3} \psi^{\prime \prime}=0
$$

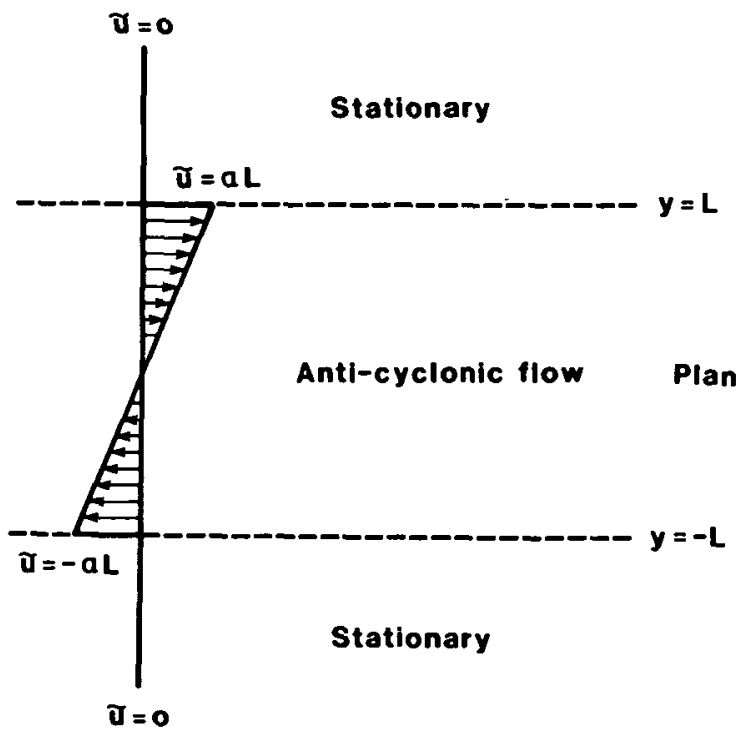

Figure 8. Flow pattern assumed in the upper layer in mid-ocean. Notice that at $y= \pm L$ there is a strong gradient in the shear. 
Assuming $\psi^{\prime \prime}$ is proportional to $\exp (l Y)$ where $l$ is a constant as required for decay away from the anti-cyclonic region, this gives

$$
l=l_{ \pm}=\frac{1}{2}\left(P_{1} \pm\left(P_{1}^{2}+4 P_{3}\right)^{1 / 2}\right) .
$$

It follows that the appropriate boundary conditions on the wave within the anti-cyclonic region are

$$
\frac{\left(d \psi^{\prime \prime} / d^{\prime} Y\right)}{\psi^{\prime \prime}}=\left\{\begin{array}{ll}
i_{+} & \text {at } Y=-Y_{B} \\
l_{-} & \text {at } Y=+Y_{B}
\end{array}\right\}
$$

As in the previous section, we integrate (4.1) from $Y=-Y_{B}$ to $Y=Y_{B}$ using a Runge-Kutta procedure. The resulting dispersion relation, with a reference frame moving with the velocity associated with the far field (or the centre of the shear) is shown in Figure 9. The behaviour shown in Figure 9 is very similar to that in Figure 5, although the wave in the present example appears to be able to travel faster than any particle within the basic shear flow. This emphasises that although the wave is strongly influenced by advection it is a freely propagating solution. The strong influence of the boundary conditions on the speed of the wave is not surprising. This type of influence is common, see for example Hughes [4]. Here the modification of the boundaries means that the wave is hybrid between a shear wave (propagating on the gradient of the shear) and an anti-cyclonic shear wave. Anti-cyclonic flows other than those shown in Figures 1 and 8 give similar overall properties for the dispersion relation.

In the introduction, it was noted that the wave being studied here is merely a new member of the vorticity class of waves. Although such waves are commonly hybrid, it is natural to ask: when is a mid-ocean wave of this class of waves best described as either a Rossby wave (the commonly assumed description) or an

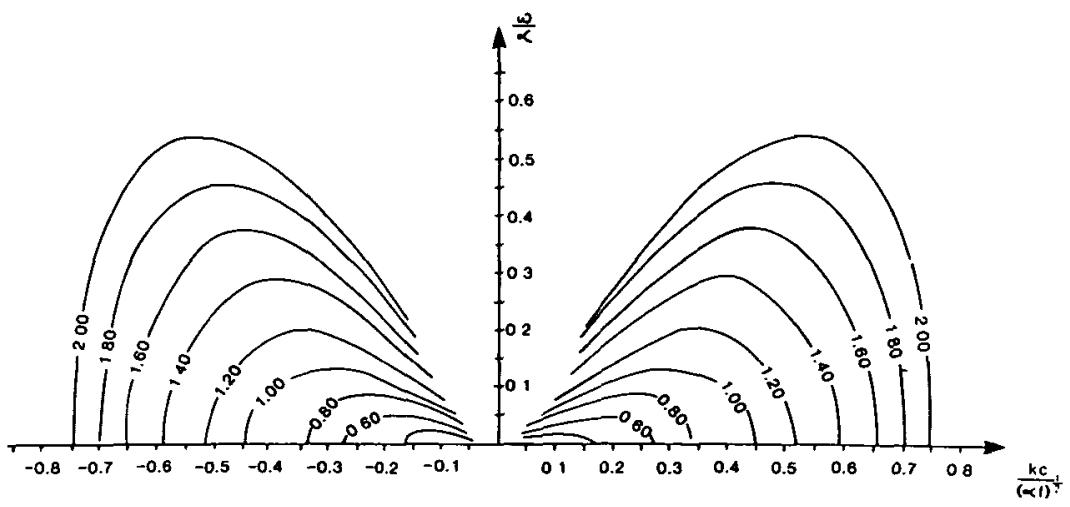

Figure 9. As for Figure 5 but using the flow structure of Figure 8. 
anti-cyclonic shear wave? From simple scaling means it is possible to show that for large amplitude waves,

$$
R=\left|\frac{\beta c^{2} / f^{2}}{c \alpha /(\alpha f)^{1 / 2}}\right|=\left|\frac{\beta c}{\alpha^{1 / 2} f^{3 / 2}}\right|
$$

is the relevant parameter. If $R \ll 1$ and $f \alpha>0$ an anti-cyclonic shear wave description is the more applicable, while if $R \gg 1$ or $f \alpha<0$ a Rossby wave description is the more appropriate. There are substantial regions of anti-cyclonic flow where one of the two descriptions is more appropriate. The condition in the case of small amplitude waves favours a Rossby wave description in all practical cases. In such cases

$$
R=\left|\frac{\beta c}{\alpha f}\right| \text {. }
$$

Why has an anti-cyclonic shear wave not been identified as a new type of wave in numerical models of the ocean circulation? The answer to this can only be speculative. Maybe it is because the numerically generated waves are commonly hybrid and any failure of the numerical model to agree with the Rossby wave dispersion relation could easily be explained by advection. In any case a model exhibiting anti-cyclonic shear waves would be required to be non-linear and possibly include a passive layer.

\section{Conclusions}

By considering the influence of vortex stretching in response to Bernoulli induced pressure variations, the class of vorticity waves has been extended. The new first baroclinic mode wave which is responsible for this extension represents a sequence of cyclonic and anti-cyclonic eddies propagating on an anti-cyclonic shear at some speed characteristic of the flow itself. These waves are limited to both low frequency and low wavenumbers. Crucial to the existence of these waves is the existence of a deep passive layer.

As to the role of these waves in the ocean, we can at present only speculate. In an oceanic anti-cyclonic shear characteristic of large scale motion, a wave of the vorticity class is likely to be hybrid between a Rossby wave and an anti-cyclonic shear wave with properties intermediate between these two waves. If the ocean has an anti-cyclonic vorticity significantly stronger than $f^{3} / \beta^{2} c^{2}$ then an anti-cyclonic shear wave may be an appropriate description with wave activity being largely trapped to the anti-cyclonic region. Wyrtki [6] has given data for the annual mean dynamic height and its variability over much of the Pacific Ocean. 
The western boundary currents are regions of both high anti-cyclonic vorticity and oceanic variability. However, this is because these regions are sources of eddy activity rather than because energy is trapped within them. More interestingly for present purposes, there appears to be a slightly higher variability in the region $10^{\circ} \mathrm{N}-35^{\circ} \mathrm{N}$ than at $35^{\circ} \mathrm{N}-60^{\circ} \mathrm{N}$ throughout the mid-ocean. Because of the slow group velocities of Rossby waves at these latitudes and the slow advective velocities in mid-ocean, it may seem unlikely that the variability in mid-ocean could have originated from the boundaries, despite the common assumption that it does. However, a closer look at the situation shows that an anti-cyclonic shear wave rather than a Rossby wave is a more appropriate description of any vorticity class wave in the region $10^{\circ} \mathrm{N}-35^{\circ} \mathrm{N}$. Furthermore such a wave has a group velocity comparable with $c$ for wavenumbers $k \sim k_{M}$. This large group velocity could enable boundary variability to extend across the Pacific Ocean as is apparently observed. More work is needed if the true role (if any) of anti-cyclonic shear waves in the ocean is to be understood.

\section{Acknowledgements}

The author is grateful to Mrs. M. Baxter, Mrs. J. Couture, and Miss G. Burt for helping in the preparation of this manuscript.

\section{References}

[1] M. Abramowitz and I. A. Stegun (eds.), Handbook of mathematical functions (Dover, New York, 1970).

[2] I. L. Collings and R. Grimshaw, "The effect of topography on the stability of a baroclinic coastal current", Dynam. Atmos. Oceans 4 (1980), 83-106.

[3] R. L. Hughes, "A solution technique for deep baroclinic rotating flows", Dynam. Atmos. Oceans 5 (1981), 159-173.

[4] R. L. Hughes, "On inertial instability of the equatorial undercurrent", Tellus 33 (1981), 291-300.

[5] P. P. Niiler and L. A. Mysak, "Barotropic waves along an eastern continental shelf", Geophys. Fluid Dynam. 2 (1971), 273-288.

[6] K. Wyrtki, "Fluctuations of dynamic topography in the Pacific Ocean", J. Phys. Oceanogr. 5 (1975), 450-459. 